\title{
Assessment of Different Quantum Mechanical Methods for the Prediction of Structure and Cohesive Energy of Molecular Crystals
}

\author{
Michele Cutini, ${ }^{\mathrm{a}}$ Bartolomeo Civalleri, ${ }^{\mathrm{a}}{ }^{*}$ Marta Corno, ${ }^{\mathrm{a}}$ Roberto Orlando, ${ }^{\mathrm{a}}$ Jan Gerit Brandenburg ${ }^{\mathrm{b}}$, \\ Lorenzo Maschio, ${ }^{\mathrm{a}}$ and Piero Ugliengo ${ }^{\mathrm{a}, *}$ \\ ${ }^{a}$ University of Turin, Department of Chemistry and NIS (Nanostructured Interfaces and Surfaces) \\ Center, Via P. Giuria 7, 10125 Turin, Italy \\ ${ }^{b}$ Mulliken Center of Theoretical Chemistry, Institut für Physikalische und Theoretische Chemie der \\ Universität Bonn, Beringstraße 4, 53115 Bonn, Germany \\ *e-mail: bartolomeo.civalleri@unito.it, piero.ugliengo@unito.it
}

\section{Supporting information}

\section{MP2 energy calculation}

The CRYSCOR program ${ }^{1,2}$ has been used for post-HF calculations. It implements second-order Møeller plesset perturbation method up to the second order (MP2) within the local Ansatz (LMP2) as proposed by Peter Pulay in the ' $80 \mathrm{~s}^{3}$ and efficiently implemented in a number of molecular codes today ${ }^{4,5}$. The method allows for an efficient truncation of contributions to the MP2 energy based on distance criteria, thus making the method quasi-linear scaling.

The very recent implementation of Orbital-Specific Virtuals (OSV) ${ }^{6,7}$ for the definition of the virtual space has been a significant step forward in the direction of making the method black-box for the user.

In the LMP2 calculations presented here the OSV scheme has been adopted starting from excitation domains as large as the whole molecule the electron belongs to. A 6-31G basis set plus polarizations, and augmented with diffuse polarization functions (called here p-aug-6-31G(d,p)), has been adopted. Such basis set already showed good performance on molecular crystals, ${ }^{8}$ and represents a good balance between cost and accuracy. Density fitting ${ }^{9}$ and multipolar approximations have been adopted, where the latter is activated for pair distances between 4 and 12 $\AA$, according to a penetration criterion. ${ }^{10}$ 
The counterpoise-corrected total LMP2 energy, is calculated as

$$
E_{\text {tot }}^{L M P 2}=E_{\text {bulk }}^{L M P 2}-E_{\text {molecule }}^{L M P 2}+E_{B S S E}^{L M P 2}
$$

Where $E_{\text {molecule }}^{L M P 2}$ is the total energy of a single molecule with geometry optimized at the B3LYP$\mathrm{D} * / \mathrm{TZP}$ level, and $E_{B S S E}^{M P 2}$ is evaluated as the difference between the total energy of a molecule in the bulk configuration, surrounded or not by a sphere of ghost atoms.

\section{Statistical functions}

MAE $=\frac{1}{N} \cdot \sum_{\mathrm{i}}\left|\delta_{\mathrm{i}}\right|, \mathbf{M A R E} \%=\frac{1}{\mathrm{~N}} \cdot \sum_{\mathrm{i}}\left|\delta_{\mathrm{i}} \%\right|, \quad \boldsymbol{\delta}_{\mathrm{i}}=\left|\mathrm{x}_{\mathrm{i}}\right|-\left|\mathrm{x}_{\mathrm{i}}^{\mathrm{exp}}\right|, \boldsymbol{\delta}_{\mathrm{i}} \%=100 \cdot \frac{\left|\mathrm{x}_{\mathrm{i}}\right|-\left|\mathrm{x}_{\mathrm{i}}^{\mathrm{exp}}\right|}{\left|\mathrm{x}_{\mathrm{i}}^{\exp }\right|} \mathbf{M E}=\frac{1}{\mathrm{~N}}$. $\sum_{\mathrm{i}} \delta_{\mathrm{i}}, \mathbf{M R E} \%=\frac{1}{\mathrm{~N}} \cdot \sum_{\mathrm{i}} \boldsymbol{\delta}_{\mathbf{i}} \%, \mathbf{S D}=\sqrt{\frac{\sum_{\mathrm{i}}\left(\boldsymbol{\delta}_{\mathrm{i}}-\overline{\boldsymbol{\delta}}\right)^{2}}{\mathrm{~N}-1}}, \mathbf{S D} \%=\sqrt{\frac{\sum_{\mathrm{i}}\left(\boldsymbol{\delta} \% \%_{\mathrm{i}}-\overline{\boldsymbol{\delta}} \%\right)^{2}}{\mathrm{~N}-1}}$, with $\mathrm{i}=1, \ldots, \mathrm{N}$.

Table S1. Computational details for the system analyzed at the HF-3c level of theory. B3LYP$\mathrm{D} * / \mathrm{TZP}$ geometry optimization were run with the same computational setup employed for the HF$3 \mathrm{c}$ calculations.

\begin{tabular}{lll}
\hline \hline Set & $\begin{array}{l}\text { Integral } \\
\text { tolerance* }\end{array}$ & Shrink factor \\
\hline X23 & 777718 & $4 * *$ \\
G60 & 777716 & 4 \\
K7- acetylene cubic/HCN/boric acid/propane & 777718 & 8 \\
K7 - acetamide/acetylene orthorhombic & 777718 & $4 * * *$ \\
K7 - ice XI & 777716 & 4 \\
Graphite & 777718 & 8 \\
$*$ The tolerance for the exchange series has set to10 $0^{-25}$ Ha for naphthalene, anthracene, benzene and propane. ** Shrink \\
factor for the $\mathrm{NH}_{3}$ case was set to $8 * * * a c e t y l e n e$ orthorhombic is calculated at the B3LYP-D*/CPC/TZP level with 8 \\
as shrink factor
\end{tabular}


Table S2. Integral tolerances (TOL) for the B3LYP-D*/TZP energy calculations on the HF-3c $\left(0.27 \mathrm{~s}_{8}\right)$ optimized structures. Shrink factor is set to 4 for all the systems, exceptions are marked with star (shrink 8).

\begin{tabular}{|c|c|c|c|c|c|}
\hline NAME & TOL & NAME & TOL & NAME & TOL \\
\hline adamantane & 777718 & dclben01 & 777716 & parbac11 & 777716 \\
\hline $\mathrm{CO}_{2}$ & 777718 & dclbqn & 777725 & phenan08 & 888840 \\
\hline cyanamide & 777718 & dimnan01 & 777725 & pymdan & 777725 \\
\hline cyc hexadione & 777718 & ditbox & 777725 & pyrene 02 & 1299960 \\
\hline cytosine & 777718 & dmbzac01 & 777725 & sucanh15 & 777716 \\
\hline ethylcarbamate & 777718 & dmnaph05 & 777725 & tatnbz & 12999100 \\
\hline $\mathrm{NH}_{3}$ & 777718 & dnbenz15 & 777725 & tchlbz & 777716 \\
\hline oxalic ac alpha & 777718 & dnitbz11 & 777725 & tcyqme & 777725 \\
\hline pyrazine & 777718 & felseu & 777725 & tepnit11 & 777725 \\
\hline pyrazole & 777718 & formac01 & 777716 & tnbenz10 & 7771250 \\
\hline succinic acid & 777718 & formamide & 777718 & tnioan & 777725 \\
\hline triazine & 777718 & hclbn 11 & 777725 & tnoxyl & 777725 \\
\hline trioxane & 777718 & imidazole & 777718 & tnphnt & 777725 \\
\hline uracil & 777718 & malnac06 & 777716 & toxocn & 777716 \\
\hline urotropine & 777718 & melami04 & 777716 & urea & 777718 \\
\hline acetic acid & 777718 & metnam04 & 777716 & wifkeb & 777716 \\
\hline $\operatorname{acrdin} 01$ & 777725 & mleica & 777716 & zzzfyw01 & 777725 \\
\hline anthracene & 999940 & mntdma01 & 777725 & zzzmuc01 & 777725 \\
\hline antquo08 & 99915100 & mtnanl & 777725 & zzzqsc01 & 777725 \\
\hline baplot01 & 777725 & naphqu & 888840 & acetamide & 777718 \\
\hline benzdc01 & 777725 & naphthalene & 777725 & acetylene cub & 777718 \\
\hline benzene & 777725 & napoac01 & 888840 & acetylene orto & 777718 \\
\hline bisjiw & 777716 & nitolu & 777725 & boric acid & 777718 \\
\hline bzamid01 & 777725 & nitpol02 & 777725 & $\mathrm{HCN}$ & 777718 \\
\hline coumar10 & 777725 & ntrgua03 & 777716 & ice XI & 777716 \\
\hline ctmtna & 777716 & ochtet12 & 777716 & propane & 777725 \\
\hline daznap & 777725 & octane 01 & 777716 & & \\
\hline dcbox 10 & 777725 & oxalic ac beta & 777718 & & \\
\hline
\end{tabular}




\section{Fitting procedure of the HF-3c dispersion term}

Graphite is a layered material in which each layer is flat with a honeycomb lattice arrangement of C atom. The interaction between planes is ruled by non covalent forces. For this reason, graphite exfoliation energy $\left(\mathrm{E}_{\mathrm{ex}}\right)$ and interlayer distance, that corresponds to half the $c$ lattice parameter, are here used as a reference value to fit dispersion term of the HF-3c method. Fully relaxed geometry and exfoliation energy were computed at different scaling of the $\mathrm{s}_{8}$ dispersion parameter. The results are reported in Table S3. We noticed that the best agreement for the geometrical part is reached by imposing $\mathrm{s}_{8}=0.27$.

Table S3. Graphite exfoliation energy and $c$ lattice parameter at different scaling of the $\mathrm{s}_{8}$ term of HF-3c method. Energy in meV per atom, length in $\AA$.

\begin{tabular}{lll}
\hline \hline Scale factors & $\mathrm{E}_{\text {exf }}$ & $c$ \\
\hline $\mathrm{S}_{8}=0.0$ & -42.8 & 6.84 \\
$\mathrm{~S}_{8}=0.25$ & -48.8 & 6.71 \\
$\mathrm{~S}_{8}=0.27$ & -49.3 & 6.70 \\
$\mathrm{~S}_{8}=0.3$ & -50.1 & 6.68 \\
$\mathrm{~S}_{8}=0.4$ & -52.8 & 6.62 \\
$\mathrm{~S}_{8}=0.5$ & -55.6 & 6.57 \\
$\mathrm{~S}_{8}=0.7$ & -60.3 & 6.50 \\
$\mathrm{~S}_{8}=1.0$ & -69.9 & 6.36 \\
Exp & $-35 /-52^{11-14}$ & $6.70^{15}$ \\
\hline \hline
\end{tabular}

\section{Fitting procedure of the $\mathrm{HF}-3 \mathrm{c}$ gCP term}

As for the D3 term of the HF-3c total energy, we also tuned the gCP correction on the graphite system. We compute the $\mathrm{E}_{\mathrm{ex}}$ curve ( $\mathrm{E}_{\mathrm{ex}} \mathrm{vs} c$ parameter) at the HF/MINIX level using both the gCP and CPC BSSE corrections. We considered the latter as a reference for the tuning procedure. While the results at the HF/MINIX/gCP level with gCP energy corrected by a scaling factor of 3.2 gives the best fitting of the HF/MINIX/CPC curve. Unfortunately, such scaling factor as applied to HF-3c method gives unsatisfactory results for the X23 set. Only for a few systems (e.g benzene, naphthalene) we obtained reasonable values for cohesive energy and cell volume. For this reason we did not use such scaling factor in the present work. Better results have been obtained when the scaling parameter is applied to both the HF and gCP energies. The best fitted scaling factor was 1.23. We then adopted such scaling factor to scale the gCP term in HF-3c for the X23 set. 
Table S4. Molecular crystals investigated in this work. References for the experimental crystal structures (crystal structure identifier) and sublimation enthalpies are reported. The values of corrected cohesive energy (see paper for further details in the correction procedure) and experimental volumes are also reported.

\begin{tabular}{|c|c|c|c|c|c|c|}
\hline CAS/COD & NAME & G60 & $\mathbf{X} 23$ & K7 & Ecoe & Vexp \\
\hline ACEMID05 & acetamide & & & $\mathrm{x}$ & -82.2 & 1474.5 \\
\hline ACETAC07 & acetic acid & $\mathrm{x}$ & $\mathrm{x}$ & & -72.8 & 297.3 \\
\hline ACETYL02 & acetylene cubic & & & $\mathrm{x}$ & $-25.2^{16}$ & 226.3 \\
\hline ACETYL04 & acetylene orthorhombic & & & $\mathrm{x}$ & $-25.2^{16}$ & 206.4 \\
\hline ACRDIN01 & acridine & $\mathrm{x}$ & & & -98.0 & 898.3 \\
\hline ADAMAN08 & adamantane & & $\mathrm{x}$ & & -69.4 & 393.1 \\
\hline ANTCEN09 & anthracene & $\mathrm{x}$ & $\mathrm{x}$ & & -112.7 & 456.5 \\
\hline ANTQUO08 & anthraquinone & $\mathrm{x}$ & & & $-115.9^{*}$ & 480.2 \\
\hline BAPLOT01 & theophyllin & $\mathrm{x}$ & & & -140.0 & 801.4 \\
\hline BENZDC01 & phthalic acid & $\mathrm{x}$ & & & -147.0 & 719.7 \\
\hline $\begin{array}{l}\text { BENZEN06 } \\
\text { Ref. }^{17} \text { and Ref. }{ }^{18} \text { a }\end{array}$ & benzene & $\mathrm{x}$ & $\mathrm{x}$ & & -55.3 & 462.5 \\
\hline BISJIW & fumaronitrile & $\mathrm{x}$ & & & -73.6 & 215.0 \\
\hline BZAMID02 & benzamide & $\mathrm{x}$ & & & -107.0 & 593.8 \\
\hline COUMAR10 & coumarin & $\mathrm{x}$ & & & -88.1 & 695.0 \\
\hline CTMTNA03 & trinitro-triazacyclohexane & $\mathrm{x}$ & & & $-117.0^{*}$ & 1588.5 \\
\hline CYANAM01 & cyanamide & & $\mathrm{x}$ & & -79.7 & 415.7 \\
\hline CYHEXO & cyclohexanedione & & $\mathrm{x}$ & & -88.6 & 279.6 \\
\hline $\begin{array}{l}\text { CYTSIN01 } \\
\text { NIST: } 71-30-7^{b}\end{array}$ & cytosine & & $\mathrm{x}$ & & -161.3 & 472.4 \\
\hline DAZNAP & phthalazine & $\mathrm{x}$ & & & -88.0 & 1342.4 \\
\hline DCBDOX10 & dichlorodioxin & $\mathrm{x}$ & & & $-114.0 \mathrm{~T}$ & 1048.6 \\
\hline DCLBEN01 & dichlorobenzene & $\mathrm{x}$ & & & -70.2 & 320.6 \\
\hline DCLBQN & dichlorobenzoquinone & $\mathrm{x}$ & & & $-74.9 *$ & 710.4 \\
\hline DIMNAN01 & nitrodimethylaniline & $\mathrm{x}$ & & & -107.7 & 389.3 \\
\hline DITBOX & dihydroanthracene & $\mathrm{x}$ & & & -99.2 & 495.8 \\
\hline DMBZAC01 & dimethylbenzoic acid & $\mathrm{x}$ & & & -109.6 & 388.7 \\
\hline DMNAPH02 & dimethylnaphthalene & $\mathrm{x}$ & & & -87.7 & 886.1 \\
\hline DNBENZ11 & dinitrobenzene & $\mathrm{x}$ & & & -92.0 & 679.7 \\
\hline DNITBZ03 & dinitrobenzene & $\mathrm{x}$ & & & -99.3 & 333.5 \\
\hline ECARBM01 & ethyl carbamate & & $\mathrm{x}$ & & -86.3 & 248.8 \\
\hline FELSEU & tetrachlorodioxin & $\mathrm{x}$ & & & $-116.3 \mathrm{~T}$ & 1195.9 \\
\hline
\end{tabular}




\begin{tabular}{|c|c|c|c|c|c|c|}
\hline FORMAC02 & formic acid & $\mathrm{x}$ & & & $-67.0 \mathrm{~T}$ & 193.0 \\
\hline Ref. $^{19}$ & formamide & $\mathrm{x}$ & $\mathrm{x}$ & & -79.2 & 217.1 \\
\hline HCLBNZ13 & hexachlorobenzene & $\mathrm{x}$ & & & $-78.3^{20}$ & 440.1 \\
\hline HXMTAM10 & hexamine & & $\mathrm{x}$ & & -86.2 & 332.4 \\
\hline IMAZOL04 & imidazole & $\mathrm{x}$ & $\mathrm{x}$ & & -86.8 & 348.8 \\
\hline JAYDUI & propane & & & $\mathrm{x}$ & $-33.5^{16} \mathrm{~T}$ & 364.9 \\
\hline MALNAC16 & malonic acid & $\mathrm{x}$ & & & -116.4 & 206.2 \\
\hline MELAMI06 & melamine & $\mathrm{x}$ & & & -125.0 & 517.3 \\
\hline METNAM08 & dimethylnitramine & $\mathrm{x}$ & & & -74.9 & 204.4 \\
\hline MLEICA01 & maleic anhydride & $\mathrm{x}$ & & & -73.8 & 413.2 \\
\hline MNTDMA01 & dimethylnitroaniline & $\mathrm{x}$ & & & -97.7 & 812.4 \\
\hline MTNANL & tetryl & $\mathrm{x}$ & & & -138.8 & 1101.5 \\
\hline NAPHQU01 & naphthoquinone & $\mathrm{x}$ & & & -96.0 & 723.2 \\
\hline NAPHTA31 & naphthalene & $\mathrm{x}$ & $\mathrm{x}$ & & -81.7 & 340.4 \\
\hline NAPOAC01 & naphthoic acid & $\mathrm{x}$ & & & -118.6 & 820.9 \\
\hline NITOLU & nitrotoluene & $\mathrm{x}$ & & & -79.8 & 1416.8 \\
\hline NITPOL02 & nitrophenol & $\mathrm{x}$ & & & $-103.8^{16 *}$ & 598.5 \\
\hline NTRGUA03 & nitroguanidine & $\mathrm{x}$ & & & -147.7 & 1578.2 \\
\hline OCHTET13 & octogen & $\mathrm{x}$ & & & $-166.9 \mathrm{~T}$ & 501.4 \\
\hline OCTANE01 & n-octane & $\mathrm{x}$ & & & $-73.1 \mathrm{~T}$ & 209.4 \\
\hline OXALAC05 & oxalic acid alpha & & $\mathrm{x}$ & & -96.3 & 307.0 \\
\hline OXALAC10 & oxalic acid beta & $\mathrm{x}$ & $\mathrm{x}$ & & -96.1 & 154.5 \\
\hline PARBAC03 & parabanic acid & $\mathrm{x}$ & & & 124.4 & 435.1 \\
\hline PHENAN08 & phenanthrene & $\mathrm{x}$ & & & -97.1 & 484.4 \\
\hline PYMDAN & pyromellitic dianhydride & $\mathrm{x}$ & & & -127.3 & 863.3 \\
\hline PYRAZI01 & pyrazine & & $\mathrm{x}$ & & -61.3 & 203.6 \\
\hline PYRENE03 & pyrene & $\mathrm{x}$ & & & $-105.3^{21}$ & 1022.9 \\
\hline PYRZOL27 & pyrazole & & $\mathrm{x}$ & & -77.7 & 703.3 \\
\hline $\operatorname{Ref}^{22}$ & ammonia & & $\mathrm{x}$ & & -37.2 & 128.6 \\
\hline $\operatorname{Ref}^{23}$ & boric acid & & & $\mathrm{x}$ & $-106.7^{24} *$ & 262.9 \\
\hline $\operatorname{Ref}^{25}$ & carbon dioxide & & $\mathrm{x}$ & & -28.4 & 177.9 \\
\hline $\operatorname{Ref}^{26}$ & hydrogen cyanide & & & $\mathrm{x}$ & $-40.6 \mathrm{~T}$ & 86.9 \\
\hline $\operatorname{Ref}^{27}$ & ice XI & & & $\mathrm{x}$ & $-59.2^{16}$ & 255.3 \\
\hline SUCACB08 & succinic acid & & $\mathrm{x}$ & & -130.3 & 241.7 \\
\hline SUCANH12 & succinic anhydride & $\mathrm{x}$ & & & -85.7 & 422.0 \\
\hline TATNBZ & triamino-trinitrobenzene & $\mathrm{x}$ & & & $-173.0 \mathrm{~T}$ & 442.5 \\
\hline
\end{tabular}




\begin{tabular}{|c|c|c|c|c|c|}
\hline TCHLBZ03 & trichlorobenzene & $\mathrm{x}$ & & $-59.2 *$ & 694.4 \\
\hline TCYQME & tetracyanoquinodimethane & $\mathrm{x}$ & & -110.0 & 1019.5 \\
\hline TEPNIT11 & dicyanobenzene & $\mathrm{x}$ & & -94.7 & 165.6 \\
\hline TNBENZ11 & trinitrobenzene & $\mathrm{x}$ & & $-112.0 *$ & 3274.0 \\
\hline TNIOAN01 & trinitroaniline & $\mathrm{x}$ & & $-130.3^{*}$ & 827.9 \\
\hline TNOXYL01 & trinitroxylene & $\mathrm{x}$ & & $-135.0 \mathrm{~T}$ & 987.1 \\
\hline TNPHNT & trinitrophenetole & $\mathrm{x}$ & & $-125.5 \mathrm{~T}$ & 1096.3 \\
\hline TOXOCN & tetroxocane & $\mathrm{X}$ & & -84.6 & 553.1 \\
\hline TRIZIN01 & triazine phase I & & $\mathrm{x}$ & -61.7 & 586.8 \\
\hline $\operatorname{Ref}^{28}$ & triazine phase II & & $\mathrm{x}$ & -61.7 & $367.4^{29,30}$ \\
\hline TROXAN11 & trioxane & & $\mathrm{x}$ & -66.4 & 616.5 \\
\hline URACIL01 & uracil & & $\mathrm{x}$ & -135.7 & 463.4 \\
\hline UREAXX12 & urea & $\mathrm{x}$ & $\mathrm{x}$ & -102.5 & 145.1 \\
\hline WIFKEB01 & dimethylurea & $\mathrm{x}$ & & -98.5 & 453.1 \\
\hline ZZZFYW02 & dinitrobenzene & $\mathrm{x}$ & & -100.5 & 680.0 \\
\hline ZZZMUC09 & trinitrotoluene & $\mathrm{x}$ & & $-118.2^{*}$ & 1770.6 \\
\hline ZZZQSC01 & dinitrotoluene & $\mathrm{x}$ & & $-103.3 \mathrm{~T}$ & 769.0 \\
\hline \multicolumn{6}{|c|}{ Sublimation enthalpies in $\operatorname{Ref}^{31}$ if not specified otherwise. } \\
\hline \multicolumn{6}{|c|}{ Experimental sublimation enthalpies at $298.15 \mathrm{~K}$, exceptions are marked with $\mathrm{T}$. } \\
\hline \multicolumn{6}{|c|}{${ }^{a}$ Cohesive energy from Ref. ${ }^{17}$. Sublimation enthalpy from Ref. ${ }^{18}$. } \\
\hline \multicolumn{6}{|c|}{${ }^{\mathrm{b}}$ Experimental sublimation enthalpies from NIST code: 71-30-7. Back correction for cohesive energy from } \\
\hline \multicolumn{6}{|c|}{ Tkatchenko's work ${ }^{18}$. } \\
\hline
\end{tabular}


Table S5. X23 set. Name, type, label (L) and cohesive energy at the LMP2/p-aug-6-31G(d,p) (MP2), HF-3c, HF-3c ( $\mathrm{s}_{8}$ 0.7) (i.e. S-HF-3c), HF-3c ( $\mathrm{s}_{8}$ 0.5) $\left(\mathrm{s}_{8} 0.5\right.$ on the table), HF-3c $\left(\mathrm{s}_{8} 0.27\right)$ ( $\mathrm{s}_{8} 0.27$ on the table), HF-3c (gCP 1.23) (gCP 1.23 on the table), SP-B3LYP-D* (SP) and B3LYPD*/TZP (B3LYP) level of theory compared with the experimental reference data (EXP).

\begin{tabular}{|c|c|c|c|c|c|c|c|c|c|c|c|}
\hline NAME & TYPE & $\mathrm{L}$ & MP2 & HF-3c & S-HF-3c & $\mathrm{s}_{8} 0.5$ & $s_{8} 0.27$ & $\mathrm{gCP} 1.23$ & SP & B3LYP & EXP \\
\hline adamantane & disp & 1 & -59.4 & -81.9 & -71.2 & -64.9 & -58.1 & -76.7 & -70.5 & -70.89 & -69.4 \\
\hline anthracene & disp & 2 & -127 & -131.4 & -113.9 & -103.2 & -92.1 & -125.7 & -97.1 & -94.76 & -112.7 \\
\hline benzene & disp & 3 & -57.7 & -65.3 & -56.4 & -51.1 & -45.4 & -62.6 & -46.5 & -46.24 & -55.3 \\
\hline $\mathrm{CO}_{2}$ & disp & 4 & -22.7 & -34.1 & -30.4 & -27.9 & -25.5 & -29.9 & -25 & -25.36 & -28.4 \\
\hline cyc. hexadione & disp & 5 & -79.8 & -84.1 & -75.4 & -70.2 & -64.6 & -77.1 & -85 & -89.13 & -88.6 \\
\hline naphthalene & disp & 6 & -91.5 & -99 & -85.5 & -77.3 & -68.8 & -94.5 & -69.4 & -69.53 & -81.7 \\
\hline pyrazine & disp & 7 & -87.6 & -74 & -65.8 & -60.6 & -55.2 & -70.4 & -59.1 & -58.86 & -61.3 \\
\hline triazine & disp & 8 & -58.9 & -65.4 & -58 & -52.9 & -48 & -61.9 & -55.6 & -55.59 & -61.7 \\
\hline trioxane & disp & 9 & -54 & -66.9 & -60.5 & -56.6 & -52.4 & -59.7 & -64.4 & -65.27 & -66.4 \\
\hline urotropine & disp & 10 & -76.7 & -103.9 & -93.9 & -87.6 & -80.7 & -97.9 & -95.3 & -94.2 & -86.2 \\
\hline acetic acid & $\mathrm{h}$ bond & 11 & -61.2 & -71.3 & -66.4 & -63.4 & -59.8 & -64.8 & -68.1 & -69.95 & -72.8 \\
\hline cyanamide & $\mathrm{h}$ bond & 12 & -77.8 & -75.2 & -70 & -66.7 & -63.1 & -71.7 & -83.1 & -83.94 & -79.7 \\
\hline ethylcarbamate & $\mathrm{h}$ bond & 13 & -73.7 & -82.9 & -76.2 & -72.1 & -67.7 & -75.6 & -82.7 & -85.58 & -86.3 \\
\hline formamide & $\mathrm{h}$ bond & 14 & -74.5 & -73.9 & -69 & -65.9 & -62.4 & -67.8 & -75.8 & -77.66 & -79.2 \\
\hline $\mathrm{NH}_{3}$ & $\mathrm{~h}$ bond & 15 & -34.1 & -42.4 & -39.2 & -37.3 & -35.1 & -39.4 & -39.2 & -38.91 & -37.2 \\
\hline oxalic ac. alpha & $\mathrm{h}$ bond & 16 & -84.2 & -117.6 & -110.2 & -105.5 & -100.5 & -105.5 & -101.5 & -101.4 & -96.3 \\
\hline oxalic ac. beta & $\mathrm{h}$ bond & 17 & -83.5 & -113 & -105.7 & -101.2 & -96.5 & -100.8 & -99.2 & -100.3 & -96.1 \\
\hline succinic ac. & $\mathrm{h}$ bond & 18 & -106.6 & -126.5 & -116.4 & -110.3 & -103.7 & -112.1 & -119.9 & -124.3 & -130.3 \\
\hline urea & $\mathrm{h}$ bond & 19 & -99.7 & -101 & -94.7 & -90.9 & -86.5 & -92.9 & -105.5 & -108.64 & -102.5 \\
\hline cytosine & mix & 20 & -172.9 & -163.1 & -151.3 & -144.1 & -136 & -153.7 & -153.7 & -156 & -161.4 \\
\hline imidazole & $\operatorname{mix}$ & 21 & -88.6 & -98.8 & -92.1 & -87.8 & -83.3 & -94.9 & -86.1 & -86.95 & -86.8 \\
\hline pyrazole & mix & 22 & -79.7 & -82.2 & -75.7 & -71.7 & -67.6 & -78.4 & -74.4 & -74.36 & -77.7 \\
\hline uracil & $\operatorname{mix}$ & 23 & -137.2 & -131.8 & -122.1 & -115.9 & -109.3 & -122.2 & -130.4 & -133.96 & -135.7 \\
\hline
\end{tabular}


Table S6. $\boldsymbol{\delta}_{\mathbf{i}} \%$ of the computed volumes at the HF-3c, S-HF-3c, and HF-3c ( $\left.s_{8} 0.27\right)$ level of theory for the G60 set.

\begin{tabular}{|c|c|c|c|}
\hline NAME & HF-3c & S-HF-3c & HF-3c $\left(\mathrm{s}_{8} 0.27\right)$ \\
\hline acetac01 & -5.2 & -2.7 & 1.2 \\
\hline $\operatorname{acrdin} 01$ & -11.0 & -8.0 & -3.2 \\
\hline antcen 14 & -10.1 & -7.1 & -2.3 \\
\hline antquo08 & -9.7 & -6.8 & -2.4 \\
\hline baplot01 & -5.9 & -3.7 & -0.1 \\
\hline benzdc01 & -8.4 & -5.3 & -0.5 \\
\hline benzen07 & -10.9 & -7.5 & -2.1 \\
\hline bisjiw & -17.2 & -14.2 & -9.4 \\
\hline bzamid01 & -9.0 & -5.9 & -1.2 \\
\hline coumar10 & -10.1 & -7.4 & -3.1 \\
\hline ctmtna & 1.8 & 4.4 & 8.4 \\
\hline daznap & -10.7 & -7.7 & -3.4 \\
\hline dcbox 10 & -6.1 & -2.4 & 3.2 \\
\hline dclben01 & -5.9 & -2.0 & 4.6 \\
\hline dclbqn & -3.4 & -0.4 & 4.2 \\
\hline dimnan01 & -4.3 & -1.3 & 3.8 \\
\hline ditbox & -13.2 & -10.3 & -5.9 \\
\hline dmbzac01 & -11.4 & -8.6 & -4.0 \\
\hline dmnaph05 & -11.1 & -8.3 & -3.8 \\
\hline dnbenz15 & -2.9 & -0.2 & 4.0 \\
\hline dnitbz11 & -4.1 & -1.6 & 2.3 \\
\hline felseu & -1.9 & 1.5 & 7.2 \\
\hline formac02 & -7.5 & 0.4 & 8.6 \\
\hline formam & -13.2 & -6.5 & 2.4 \\
\hline hclbn11 & 0.0 & 3.8 & 10.2 \\
\hline imazol13 & -9.7 & -6.8 & -2.1 \\
\hline malnac06 & -2.6 & 0.0 & 4.5 \\
\hline melami04 & -6.4 & -4.0 & -0.3 \\
\hline metnam04 & -0.1 & 2.8 & 7.5 \\
\hline mleica & -7.2 & -4.4 & -0.1 \\
\hline mntdma01 & -6.8 & -4.1 & 0.4 \\
\hline mtnanl & -4.2 & -1.9 & 2.2 \\
\hline naphqu & -8.3 & -5.2 & -0.2 \\
\hline naphta10 & -10.3 & -7.1 & -2.0 \\
\hline napoac01 & -11.8 & -9.1 & -4.9 \\
\hline nitolu & -11.0 & -8.1 & -3.7 \\
\hline nitpol02 & -6.3 & -3.1 & 1.8 \\
\hline ntrgua03 & -6.2 & -3.6 & 0.5 \\
\hline ochtet12 & 4.2 & 6.9 & 11.1 \\
\hline octane 01 & -12.4 & -9.5 & -4.9 \\
\hline
\end{tabular}




\begin{tabular}{|l|r|r|r|} 
oxalac04 & -4.5 & -2.0 & 2.2 \\
\hline parbac11 & -2.2 & 0.1 & 3.4 \\
\hline phenan08 & -12.4 & -9.6 & -5.2 \\
\hline pymdan & -7.5 & -5.2 & -1.4 \\
\hline pyrene02 & -9.8 & -7.2 & -3.1 \\
\hline sucanh15 & -4.7 & -1.9 & 2.4 \\
\hline tatnbz & -4.5 & -1.6 & 3.2 \\
\hline tchlbz & -0.2 & 4.3 & 11.5 \\
\hline tcyqme & -8.4 & -6.1 & -2.6 \\
\hline tepnit11 & -13.9 & -11.0 & -6.5 \\
\hline tnbenz10 & -2.7 & 0.1 & 4.0 \\
\hline tnioan & -1.2 & 1.2 & 4.5 \\
\hline tnoxyl & -6.6 & -4.1 & -0.4 \\
\hline tnphnt & -5.1 & -2.9 & 0.4 \\
\hline toxocn & -10.7 & -8.3 & -4.4 \\
\hline ureaxx12 & -5.0 & -3.2 & -0.8 \\
\hline wifkeb & -7.5 & -4.8 & -0.7 \\
\hline zzzfyw01 & -4.9 & -1.9 & 2.6 \\
\hline zzzmuc01 & -6.5 & 0.6 & 4.5 \\
\hline zzzqsc01 & -3.4 & \\
\hline
\end{tabular}


Table S7.Name, type and $\boldsymbol{\delta}$ of the cohesive energy at the HF-3c, S-HF-3c, SP-B3LYP-D* and B3LYP-D*/CPC/6-31G* level of theory for the G60 set.

\begin{tabular}{|c|c|c|c|c|c|c|}
\hline NAME & TYPE & HF-3c & S-HF-3c & SP-B3LYP-D* & B3LYP/CPC/6-31G* & EXP \\
\hline acetac 01 & $\mathrm{~h}$ bond & -1.5 & -6.4 & -4.7 & 2.9 & -72.8 \\
\hline $\operatorname{acrdin} 01$ & disp & 28.6 & 11.3 & -5.3 & -8.4 & -98 \\
\hline antcen14 & disp & 18.7 & 1.1 & -15.6 & -19.6 & -112.7 \\
\hline antquo08 & disp & 17.7 & 0.6 & -4.2 & -20 & -115.9 \\
\hline baplot01 & $\operatorname{mix}$ & 2.4 & -9.7 & -8.7 & -6.6 & -140 \\
\hline benzdc01 & $\operatorname{mix}$ & 6.2 & -6.9 & -9.4 & 17 & -147 \\
\hline benzen07 & disp & 10.3 & 1.4 & -8.8 & -7.2 & -55.3 \\
\hline bisjiw & disp & 8.4 & -0.4 & -5.9 & -6.8 & -73.6 \\
\hline bzamid01 & $\operatorname{mix}$ & 11.9 & 0.3 & -3.6 & 0.7 & -107 \\
\hline coumar10 & disp & 13.9 & 2.2 & 1 & -0.3 & -88.1 \\
\hline ctmtna & disp & -6 & -15.8 & 9.2 & 13 & -117 \\
\hline daznap & disp & 14.4 & 3 & 0 & -2.2 & -88 \\
\hline dcbox10 & disp & -4.7 & -21.4 & -25.7 & -36.7 & -114 \\
\hline dclben01 & disp & -5 & -14.6 & -18.7 & -17.5 & -70.2 \\
\hline dclbqn & disp & 5.3 & -4.1 & -8.4 & -6.5 & -74.9 \\
\hline dimnan01 & disp & 1.9 & -10.5 & -4.5 & 1.8 & -107.7 \\
\hline ditbox & disp & 25.5 & 8.9 & -5.4 & -7.6 & -99.2 \\
\hline dmbzac01 & $\operatorname{mix}$ & 13.5 & 0.6 & -5.3 & 3 & -109.6 \\
\hline dmnaph05 & disp & 22.7 & 8.4 & -2.9 & -4.4 & -87.7 \\
\hline dnbenz15 & disp & -3.6 & -12.9 & -7.5 & -4.6 & -92 \\
\hline dnitbz11 & disp & -4.8 & -14.7 & -11.9 & -14.4 & -99.3 \\
\hline felseu & disp & -0.6 & -18.2 & -22.7 & -16.9 & -116.3 \\
\hline formac02 & $\mathrm{h}$ bond & -3.7 & -7.7 & -6.6 & 3.6 & -67 \\
\hline formam & $\mathrm{h}$ bond & -5.3 & -10.2 & -3.4 & 4.9 & -79.2 \\
\hline hclbn11 & disp & 18 & 1.8 & -15.5 & -16.5 & -78.3 \\
\hline imazol13 & $\operatorname{mix}$ & 12 & 5.3 & -0.7 & 4 & -86.8 \\
\hline malnac06 & $\mathrm{h}$ bond & -3.1 & $\begin{array}{l}-10.8 \\
\end{array}$ & -5.3 & 16.4 & -116.4 \\
\hline melami04 & $\operatorname{mix}$ & 34.1 & 21 & 13.2 & 25.6 & -125 \\
\hline metnam04 & disp & -10.1 & -16.3 & -0.9 & -2.4 & -74.9 \\
\hline mleica & disp & 1.1 & $\begin{array}{l}-5.6 \\
\end{array}$ & -6.6 & -5 & $\begin{array}{ll}-73.8 \\
\end{array}$ \\
\hline mntdma01 & disp & 4.2 & -7.3 & -3.3 & -1.6 & -97.7 \\
\hline mtnanl & disp & -19.5 & -31.2 & -18.3 & -10.6 & -138.8 \\
\hline naphqu & disp & 8.5 & -5 & -7.4 & -4.5 & -96 \\
\hline naphta10 & disp & 17.3 & 3.8 & -12.3 & -12.6 & -81.7 \\
\hline napoac01 & mix & 18.8 & 4.1 & -8.4 & -6.6 & -118.6 \\
\hline nitolu & disp & 5.5 & -4.1 & -5.7 & -6.1 & -79.8 \\
\hline nitpol02 & $\operatorname{mix}$ & 3.8 & -6 & -3.7 & 2.4 & -103.8 \\
\hline ntrgua 03 & $\operatorname{mix}$ & -12.7 & -21.5 & -9.1 & 2.5 & -147.7 \\
\hline ochtet12 & disp & -27.8 & -39.6 & -7.8 & 0.6 & -166.9 \\
\hline octane 01 & disp & 15 & 3.4 & -5.7 & -7.8 & \begin{tabular}{|l|}
-73.1 \\
\end{tabular} \\
\hline oxalac04 & $\mathrm{h}$ bond & 16.9 & 9.6 & 3.1 & 32.4 & -96.1 \\
\hline parbac11 & $\operatorname{mix}$ & -10.5 & -17.9 & -11.9 & -7.6 & -124.4 \\
\hline phenan08 & disp & 23.4 & 7.6 & -7.2 & -9 & $\begin{array}{l}-97.1 \\
\end{array}$ \\
\hline pymdan & disp & 1.3 & -10.7 & -15 & -15.9 & -127.3 \\
\hline pyrene 02 & disp & 25.1 & 8 & -8 & -11.4 & -105.3 \\
\hline sucanh15 & disp & 0.6 & -6.9 & -1.8 & 3.9 & -85.7 \\
\hline tatnbz & disp & -41.2 & -57 & -21 & -19.5 & -173 \\
\hline tchlbz & disp & 8.4 & -2.6 & -8.3 & -5.9 & -59.2 \\
\hline tcyqme & disp & 39.1 & 23.6 & 7.3 & 4.8 & -110 \\
\hline tepnit11 & disp & 15.6 & 3.2 & -8.6 & -10.5 & -94.7 \\
\hline tnbenz10 & disp & -15.8 & -25.2 & -16.7 & -18.8 & -112 \\
\hline tnioan & $\operatorname{mix}$ & -23 & -33.9 & -21 & -21.5 & -130.3 \\
\hline tnoxyl & disp & -16.3 & -28.5 & -19.3 & -25.6 & -135 \\
\hline tnphnt & disp & -13.9 & -24.7 & -8.9 & -13.5 & -125.5 \\
\hline toxocn & disp & 12.7 & 4.2 & -3.2 & 0.4 & -84.6 \\
\hline
\end{tabular}




\begin{tabular}{|l|l|l|l|l|l|l|}
\hline ureaxx12 & h bond & -1.6 & -7.9 & 3 & 17 & -102.5 \\
\hline wifkeb & mix & 5.1 & -3.5 & 4.1 & 8.5 & -98.5 \\
\hline Zzzfyw01 & disp & -2.4 & -12.6 & -10.1 & -11 & -100.5 \\
\hline zzzmuc01 & disp & -13.9 & -24.2 & -14.7 & -15.9 & -118.2 \\
\hline zzzqsc01 & disp & -8.3 & -19.2 & -13.7 & -15.6 & -103.3 \\
\hline
\end{tabular}


Table S8. Cell volumes (in $\AA^{3}$ ) and $\Delta \mathrm{E}_{\mathrm{c}}\left(\mathrm{in} \mathrm{kJ} \cdot \mathrm{mol}^{-1}\right.$ ) for the $\mathrm{K} 7$ set evaluated at the HF-3c, S-HF-3c and B3LYP-D*/TZP (B3LYP in table) levels of theory. HF-3c-(0.27 $\left.\mathrm{s}_{8}\right)$ optimized volumes and cohesive energy at the SP-B3LYP-D* level of theory are also reported. Percentage error reported in brackets.

\begin{tabular}{|c|c|c|c|c|c|c|c|c|c|c|}
\hline & & & & HF-3c-(0.27 & & & & & SP-B3LYP- & \\
\hline & $\mathrm{HF}-3 \mathrm{c}$ & S-HF-3c & B3LYP & $\left.\mathrm{s}_{8}\right)$ & EXP & $\mathrm{HF}-3 \mathrm{c}$ & S-HF-3c & B3LYP & $\mathrm{D}^{*}$ & EXP \\
\hline & \multicolumn{5}{|c|}{$\mathrm{V}$} & \multicolumn{5}{|c|}{$\Delta \mathrm{E}_{\mathrm{c}}$} \\
\hline Acetamide & $1376.0(-6.7)$ & $1411.3(-4.3)$ & $1441.6(-2.2)$ & $1462.3(-0.8)$ & 1474.5 & $-82.4(0.3)$ & $-76.5(-6.9)$ & $-87.5(6.4)$ & $-84.9(3.3)$ & -82.2 \\
\hline \multicolumn{11}{|l|}{ Acetylene } \\
\hline cubic & $174.7(-22.8)$ & $182.2(-19.5)$ & $212.8(-6.0)$ & $203.8(-9.9)$ & 226.3 & $-32.4(28.7)$ & $-28.6(13.3)$ & $-20.3(-19.5)$ & $-20.0(-20.8)$ & -25.2 \\
\hline \multicolumn{11}{|l|}{ Acetylene } \\
\hline orthorhombic & $185.4(-10.2)$ & $192.6(-6.7)$ & $202.8(-1.8)$ & $193.9(-6.1)$ & 206.4 & $-29.5(17.1)$ & $-26.2(4.0)$ & $-21.3(-15.4)$ & $-20.8(-17.5)$ & -25.2 \\
\hline & & & & & & & & & & - \\
\hline Boric acid & $229.2(-12.8)$ & $234.7(-10.7)$ & $254.7(-3.1)$ & $244.0(-7.2)$ & 262.9 & $-136.2(27.7)$ & $-129.1(21.0)$ & $-106.7(0.0)$ & $-104.8(-1.8)$ & 106.7 \\
\hline \multicolumn{11}{|l|}{ Hydrogen } \\
\hline cyanide & $70.2(-19.2)$ & $73.2(-15.8)$ & $81.8(-5.9)$ & $79.8(-8.2)$ & 86.9 & $-45.3(11.6)$ & $-41.6(2.5)$ & $-39.8(-2.1)$ & $-39.6(-2.5)$ & -40.6 \\
\hline Ice XI & $241.3(-5.5)$ & $243.2(-4.8)$ & $250.9(-1.7)$ & $245.9(-3.7)$ & 255.3 & $-68.0(14.8)$ & $-66.1(11.6)$ & $-65.2(10.1)$ & $-65.5(10.7)$ & -59.2 \\
\hline Propane & $336.2(-7.9)$ & $348.8(-4.4)$ & $358.9(-1.6)$ & $368.6(1.0)$ & 364.9 & $-35.3(5.5)$ & $-30.6(-8.6)$ & $-26.3(-21.4)$ & $-26.4(-21.1)$ & -33.5 \\
\hline MAE & 38 & 27 & 11 & 12.3 & & 8.1 & 6.2 & 4.0 & 4.1 & \\
\hline SD & 30 & 20 & 10 & 8.5 & & 9.9 & 9.2 & 5.0 & 4.7 & \\
\hline MARE\% & 12.1 & 9.5 & 3.2 & 5.3 & & 15 & 10 & 11 & 11 & \\
\hline $\mathrm{SD} \%$ & 6.6 & 6.1 & 1.9 & 4.0 & & 11 & 11 & 13 & 13 & \\
\hline
\end{tabular}


Table S9. Comparison of different dispersion schemes on computing cohesive energy for the X 23 and G60 sets. The statistical analysis is reported for the $\mathrm{D}^{*}, \mathrm{D} 3$ and $\mathrm{D} 3^{\mathrm{ABC}}$ schemes. The statistic functions are reported with the following format MAE $\pm \mathrm{SD}[\mathrm{ME}]$, reported in $\mathrm{kJ} \cdot \mathrm{mol}^{-1}$.

\begin{tabular}{|c|c|c|c|c|}
\hline Method & All & Dispersive & H-bond & Mixed \\
\hline \multicolumn{5}{|c|}{ X23 - On the B3LYP-D*/TZP optimized structures } \\
\hline B3LYP-D*/TZP & $4.6 \pm 6.0[-1.8]$ & $6.2 \pm 7.4[-4.2]$ & $3.6 \pm 4.1[1.1]$ & $2.7 \pm 2.4[-2.6]$ \\
\hline B3LYP-D3/TZP & $7.4 \pm 3.9[7.4]$ & $6.1 \pm 3.2[6.1]$ & $8.6 \pm 4.6[8.6]$ & $8.1 \pm 3.1[8.1]$ \\
\hline B3LYP-D3 ${ }^{\mathrm{ABC}} / \mathrm{TZP}$ & $4.1 \pm 4.4[-2.9]$ & $2.3 \pm 2.9[0.5]$ & $6.2 \pm 4.9[5.8]$ & $3.8 \pm 2.1[3.8]$ \\
\hline \multicolumn{5}{|c|}{$\mathrm{X} 23$ - On the HF-3c $\left(\mathrm{s}_{8} 0.27\right)$ optimized structures } \\
\hline B3LYP-D*/TZP & $5.2 \pm 5.8[-2.9]$ & $6.4 \pm 7.0[-4.4]$ & $4.3 \pm 5.2[-0.6]$ & $4.3 \pm 3.0[-4.3]$ \\
\hline B3LYP-D3/TZP & $6.5 \pm 4.0[6.4]$ & $5.9 \pm 3.5[5.9]$ & $7.0 \pm 5.3[6.9]$ & $6.8 \pm 1.8[6.8]$ \\
\hline B3LYP-D3 ${ }^{\mathrm{ABC}} / \mathrm{TZP}$ & $3.5 \pm 4.3[2.4]$ & $2.0 \pm 2.8[0.5]$ & $5.6 \pm 5.7[4.3]$ & $2.7 \pm 1.1[2.7]$ \\
\hline \multicolumn{5}{|c|}{ G60 - On the HF-3c $\left(\mathrm{s}_{8} 0.27\right)$ optimized structures } \\
\hline B3LYP-D*/TZP & $8.8 \pm 7.6[-7.5]$ & $9.6 \pm 7.4[-8.8]$ & $4.4 \pm 4.3[-2.3]$ & $8.3 \pm 8.5[-5.4]$ \\
\hline B3LYP-D3/TZP & $9.4 \pm 9.1[8.4]$ & $10.0 \pm 9.6[8.9]$ & $5.3 \pm 4.9[5.0]$ & $9.6 \pm 9.0[8.3]$ \\
\hline B3LYP-D3 ${ }^{\mathrm{ABC}} / \mathrm{TZP}$ & $6.9 \pm 8.8[2.2]$ & $7.3 \pm 9.4[2.0]$ & $3.6 \pm 4.5[2.6]$ & $7.1 \pm 8.7[2.3]$ \\
\hline
\end{tabular}


Table S10. X23, G60 and K7 summary (82 molecular crystals, MC82 set). MAE, SD and MAX of the $\Delta$ Ec evaluated at the HF-3c, S-HF-3c, HF-3c-(0.27 s8), B3LYP-D*/TZP and SP-B3LYP-D* levels of theory. The statistical analysis of the optimized volumes evaluated at the HF-3c, S-HF-3c, HF-3c-(0.27 s8) and B3LYP-D*/TZP levels of theory are also reported. The statistic functions are in the following format $\mathrm{MAE} \pm \mathrm{SD}(\mathrm{MAX})$. The cohesive energies were estimated from experimental sublimation energies by applying the $2 \mathrm{RT}$ correction factor.

\begin{tabular}{|c|c|c|c|c|c|c|c|c|}
\hline & \multicolumn{4}{|c|}{$\mathrm{V}(\%)$} & \multicolumn{4}{|c|}{$\mathrm{E}_{\mathrm{coe}}\left(\mathrm{kJ} \cdot \mathrm{mol}^{-1}\right)$} \\
\hline & $\mathrm{X} 23$ & G60 & K7 & TOT & $\mathrm{X} 23$ & G60 & K7 & TOT \\
\hline $\mathrm{HF}-3 \mathrm{c}$ & $8.0 \pm 2.5$ & $6.9 \pm 4.2$ & $12.1 \pm 6.6$ & $7.6 \pm 4.4$ & $9.2 \pm 9.4$ & $13 \pm 16$ & $8.1 \pm 9.9$ & $11 \pm 14$ \\
\hline S-HF-3c & $5.2 \pm 2.3$ & $4.7 \pm 4.2$ & $9.5 \pm 6.1$ & $5.2 \pm 4.4$ & $7.1 \pm 8.1$ & $12 \pm 15$ & $6.2 \pm 9.2$ & $11 \pm 14$ \\
\hline HF-3c- $\left(0.27 \mathrm{~s}_{8}\right)$ & $1.9 \pm 2.3$ & $3.5 \pm 4.5$ & $5.3 \pm 4.0$ & $3.4 \pm 4.4$ & $10.8 \pm 8.2$ & $22 \pm 15$ & $6.9 \pm 8.5$ & $19 \pm 15$ \\
\hline SP-B3LYP-D* & & I & I & I & $4.5 \pm 5.6$ & $8.5 \pm 7.6$ & $4.1 \pm 4.7$ & $7.4 \pm 7.7$ \\
\hline B3LYP-D*/TZP & $3.0 \pm 1.9$ & l & $3.2 \pm 1.9$ & l & $4.4 \pm 5.7$ & $10 \pm 12^{\mathrm{a}}$ & $4.0 \pm 5.0$ & l \\
\hline${ }^{a}$ data from ref. 27 & & & & & & & & \\
\hline
\end{tabular}




\section{References}

(1) Pisani, C.; Schütz, M.; Casassa, S.; Usvyat, D.; Maschio, L.; Lorenz, M.; Erba, A. Cryscor: A Program for the Post-Hartree-Fock Treatment of Periodic Systems. Phys. Chem. Chem. Phys. 2012, 14, 7615.

(2) Maschio, L. Local MP2 with Density Fitting for Periodic Systems: A Parallel Implementation. J. Chem. Theory Comput. 2011, 7, 2818-2830.

(3) Pulay, P. Localizability of Dynamic Electron Correlation. Chem. Phys. Lett. 1983, 100, 151.

(4) Riplinger, C.; Neese, F. An Efficient and near Linear Scaling Pair Natural Orbital Based Local Coupled Cluster Method. J. Chem. Phys. 2013, 138, 034106.

(5) Schütz, M.; Hetzer, G.; Werner, H.-J. Low-Order Scaling Local Electron Correlation Methods. I. Linear Scaling Local MP2. J. Chem. Phys. 1999, 111, 5691-5705.

(6) Usvyat, D.; Maschio, L.; Schütz, M. Periodic Local MP2 Method Employing Orbital Specific Virtuals. J. Chem. Phys. 2015, 143, 102805.

(7) Kurashige, Y.; Yang, J.; Chan, G. K.-L.; Manby, F. R. Optimization of Orbital-Specific Virtuals in Local Møller-Plesset Perturbation Theory. J. Chem. Phys. 2012, 136, 124106.

(8) Maschio, L.; Civalleri, B.; Ugliengo, P.; Gavezzotti, A. Intermolecular Interaction Energies in Molecular Crystals: Comparison and Agreement of Localized Møller-Plesset 2, Dispersion-Corrected Density Functional, and Classical Empirical Two-Body Calculations. J. Phys. Chem. A 2011, 115, 11179-11186.

(9) Maschio, L.; Usvyat, D. Fitting of Local Densities in Periodic Systems. Phys. Rev. B 2008, $78,1-4$.

(10) Kats, D. Speeding up Local Correlation Methods. J. Chem. Phys. 2014, 141, 244101.

(11) Girifalco, L. A.; Lad, R. A. Energy of Cohesion, Compressibility, and the Potential Energy Functions of the Graphite System. J. Chem. Phys. 1956, 25, 693.

(12) Benedict, L. X.; Chopra, N. G.; Cohen, M. L.; Zettl, A.; Louie, S. G.; Crespi, V. H. Microscopic Determination of the Interlayer Binding Energy in Graphite. Chem. Phys. Lett. 1998, 286, 490-496.

(13) Trickey, S. B.; Müller-Plathe, F.; Diercksen, G. H. F.; Boettger, J. C. Interplanar Binding and Lattice Relaxation in a Graphite Dilayer. Phys. Rev. B 1992, 45, 4460-4468.

(14) Zacharia, R.; Ulbricht, H.; Hertel, T. Interlayer Cohesive Energy of Graphite from Thermal Desorption of Polyaromatic Hydrocarbons. Phys. Rev. B - Condens. Matter Mater. Phys. 
2004, 69, 155406.

(15) Chung, D. D. L. Review: Graphite. J. Mater. Sci. 2002, 37, 1475-1489.

(16) Chickosa, J. S.; Acree, W. E. Enthalpies of Sublimation of Organic and Organometallic Compounds. 1910-2001. J. Phys. Chem. Ref. Data 2002, 31, 537-698.

(17) Yang, J.; Hu, W.; Usvyat, D.; Matthews, D.; Schütz, M.; Chan, G. K.-L. Ab Initio Determination of the Crystalline Benzene Lattice Energy to Sub-Kilojoule/mole Accuracy. Science, 2014, 345, 640.

(18) Reilly, A. M.; Tkatchenko, A. Understanding the Role of Vibrations, Exact Exchange, and Many-Body van Der Waals Interactions in the Cohesive Properties of Molecular Crystals. $J$. Chem. Phys. 2013, 139, 024705.

(19) Torrie, B. H.; O’Donovan, C.; Powell, B. M. Structure of Solid Formamide at 7 K. Mol. Phys. 1994, 82, 643.

(20) Verevkin, S. P.; Emel'yanenko, V. N.; Klamt, A. Thermochemistry of Chlorobenzenes and Chlorophenols: Ambient Temperature Vapor Pressures and Enthalpies of Phase Transitions. J. Chem. Eng. Data 2007, 52, 499-510.

(21) Roux, M. V.; Temprado, M.; Chickos, J. S.; Nagano, Y. Critically Evaluated Thermochemical Properties of Polycyclic Aromatic Hydrocarbons. J. Phys. Chem. Ref. Data 2008, 37, 1855-1996.

(22) Hewat, A. W.; Riekel, C. The Crystal Structure of Deuteroammonia between 2 and 180 K by Neutron Powder Profile Refinement. Acta Crystallogr. Sect. A Found. Crystallogr. 1979, 35, $569-571$.

(23) Gajhede, M.; Larsen, S.; Rettrup, S. Electron Density of Orthoboric Acid Determined by XRay Diffraction at $105 \mathrm{~K}$ and Ab Initio Calculations. Acta Crystallogr. Sect. B 1986, 42, 545.

(24) Chase, M. W. NIST-JANAF Themochemical Tables, Fourth Edition. J. Phys. Chem. Ref. Data 1998, 9, 1-1951.

(25) Simon, A.; Peters, K. Single-Crystal Refinement of the Structure of Carbon Dioxide. Acta Crystallogr. Sect. B Struct. Crystallogr. Cryst. Chem. 1980, 36, 2750-2751.

(26) Dulmage, W. J.; Lipscomb, W. N. The Crystal Structures of Hydrogen Cyanide, HCN. Acta Crystallogr. Sect. A 1951, 4, 330.

(27) Howe, R.; Whitworth, R. W. A Determination of the Crystal Structure of Ice XI. J. Chem. Phys. 1989, 90, 4450. 
(28) Smith, J. H.; Rae, A. I. M. The Structural Phase Change in S-Triazine. I. The Crystal Structure of the Low-Temperature Phase. J. Phys. C Solid State Phys. 1978, 11, 1761.

(29) Prasad, S. M.; Rae, A. I. M.; Hewat, A. W.; Pawley, G. S. The Crystal Structure of STriazine at 5K. J. Phys. C Solid State Phys. 1981, 14, 929-931.

(30) Citroni, M.; Fanetti, S.; Bazzicalupi, C.; Dziubek, K.; Pagliai, M.; Nobrega, M. M.; Mezouar, M.; Bini, R. Structural and Electronic Competing Mechanisms in the Formation of Amorphous Carbon Nitride by Compressing S-Triazine. J. Phys. Chem. C 2015, 119, 28560 28569.

(31) National Institute of Standards and Technology. NIST Chemistry WebBook http://webbook.nist.gov/. 